\title{
La communauté protestante d'Authon-du-Perche au XVIIe siècle : de l'apogée à la résistance
}

\section{Amélie Duchemin}

\section{(2) OpenEdition}

\section{Journals}

\section{Édition électronique}

URL : http://journals.openedition.org/abpo/827

DOI : $10.4000 / a b p o .827$

ISBN : 978-2-7535-1502-4

ISSN : 2108-6443

Éditeur

Presses universitaires de Rennes

Édition imprimée

Date de publication : 30 juin 2006

Pagination : 61-79

ISBN : 978-2-7535-0331-1

ISSN : 0399-0826

\section{Référence électronique}

Amélie Duchemin, «La communauté protestante d'Authon-du-Perche au XVIIe siècle : de l'apogée à la résistance », Annales de Bretagne et des Pays de l'Ouest [En ligne], 113-2 | 2006, mis en ligne le 30 juin 2008, consulté le 01 mai 2019. URL : http://journals.openedition.org/abpo/827 ; DOI : 10.4000/ abpo.827 


\title{
La communauté protestante d'Authon-du-Perche au XVII ${ }^{\mathrm{e}}$ siècle : de l'apogée à la résistance
}

\author{
Amélie Duchemin \\ étudiante, université du Maine ${ }^{1}$
}

Authon-du-Perche, bourg d'environ 230 feux au milieu du XvII ${ }^{\mathrm{e}}$ siècle, est situé à mi-chemin entre Le Mans et Chartres. Si la paroisse d'Authon appartient au diocèse de Chartres et à la généralité d'Orléans, sa communauté réformée dépend de la province synodale d'Île-de-France-ChampagnePicardie-Pays Chartrain ${ }^{2}$. Or, l'Église d'Authon est excentrée au sud du colloque de Beauce. Elle se trouve ainsi éloignée des autres Églises de son colloque (Chartres ou La Ferté-Vidame par exemple) et de sa province avec lesquelles elle n'entretient que peu de relations. Géographiquement, l'Église réformée d'Authon est plus proche de communautés de l'Orléanais-Berry (Dangeau, Châteaudun) ou d'Anjou-Touraine-Maine (Bellême, Le Mans ou Saint-Aignan) et c'est avec ces Églises qu'elle noue les liens les plus étroits.

Le protestantisme naît à Authon dès le milieu du Xvi ${ }^{\mathrm{e}}$ siècle, mais peu de faits sont connus sur les réformés authonais au cours de ces premières décennies. Nous en sommes presque réduits aux hypothèses. On peut tout d'abord s'appuyer sur une liste de ministres réfugiés à Londres lors de la Saint-Barthélemy. On y trouve la mention de " Michel de Montescot, ministre d'Authen ${ }^{3}$ ». Selon Henri Lehr, Michel de Montescot était ministre d'Authon depuis $1563^{4}$. Or, c'est lors de cette décennie que se sont formée les autres communautés proches d'Authon : Luigny, Brou et Dangeau ${ }^{5}$. L'hypothèse est donc concevable. François Guillon propose de son côté

1. Cet article a été rédigé à partir d'un mémoire de maîtrise soutenu en 2003 à l'Université du Maine, La Communauté protestante d'Authon-du-Perche au XVII siècle : de l'apogée à la résistance.

2. Sous le régime de l'édit de Nantes, la France protestante est divisée en 16 provinces synodales, chaque province en colloques et les colloques en Églises.

3. BONNET, Jules, " Ministres réfugiés à Londres après la Saint-Barthélemy ", Bulletin de la Société de l'Histoire du Protestantisme Français, 1853, p. 25-26.

4. LEHR, Henri, La Réforme et les églises réformées dans le département d'Eure-et-Loir (1523-1911), Paris, Librairie Fischbacher, 1912, p. 152-155.

5. LeHR, H., La Réforme et les églises réformées, op. cit., p. 153. 
une autre explication pour la naissance du protestantisme à Authon ${ }^{6}$. Selon lui, la Réforme aurait été importée par Bertrand Robethon, médecin d'Henri III, à l'extrême fin du XVI ${ }^{\mathrm{e}}$ siècle. Il est en fait envisageable d'imaginer une première création d'une Église calviniste à Authon vers 1563, création mise à mal par la persécution de la Saint-Barthélemy en 1572. Les réformés se sont peut-être alors rendus au prêche à Dangeau, à trente kilomètres à l'est d'Authon, avant l'arrivée vers 1598, ou peut-être avant, d'un nouveau pasteur parmi une population protestante de notables, tels les Robethon. Le ministre Jacques Couronné permet alors à la communauté de renaître ${ }^{7}$. C'est en tout cas à partir de 1598 qu'ont été conservés les registres d'état civil de l'Église calviniste. C'est principalement grâce à ces registres que nous apparaît la communauté réformée d'Authon.

\section{La communauté réformée}

C'est par cette principale source que s'appréhende le plus facilement la communauté huguenote. Le registre de l'état civil est tenu par les pasteurs de 1598 à 1683 . Il se compose de seize cahiers : le premier comprend les actes enregistrés entre 1598 et 1668; par la suite, il existe un cahier par année, de 1669 à 1683. Seul le cahier de l'année 1677 a été perdu. Si tous les baptêmes sont enregistrés au cours du siècle, les mariages sont, eux, transcrits avec beaucoup moins de soin. Quant aux décès, ils sont pratiquement inexistants avant $1668^{8}$. Par ces éléments démographiques, l'évolution de la population, la culture religieuse et les caractéristiques sociales de cette communauté réformée peuvent être successivement étudiées.

Les registres d'état civil permettent de comptabiliser 364 couples calvinistes, soit 1451 personnes sur un siècle. Or, en 1665, la paroisse devait compter 229 feux $^{9}$, soit 1030 personnes, toutes confessions confondues. L'archidiacre du Dunois, en 1670, recense 650 communiants ${ }^{10}$; il reste donc 380 protestants. On obtient alors approximativement la proportion de catholiques et de réformés : $63 \%$ des Authonais seraient donc catholiques pour $37 \%$ de calvinistes.

6. GuILLON, François, "Le protestantisme à Authon-du-Perche et dans les environs ", Mémoires de la Société Archéologique d'Eure-et-Loir, tome XIV, 1905, p. 167.

7. Le premier baptême enregistré par Jacques Couronné date du 20 février 1598. Toutefois, rien ne permet d'affirmer qu'il n'y avait pas un pasteur avant Jacques Couronné, d'autant que le statut de l'Église d'Authon reste incertain. Ce n'est pas une Église de fief puisque le seigneur est catholique; ce n'est pas une Église de concession, puisqu'en raison des articles secrets de l'Édit de Nantes, il ne doit y avoir qu'un lieu de culte par bailliage et ce lieu est fixé à Orléans. Demeure le culte de possession, c'est-àdire un exercice autorisé parce qu'il existait au cours des années 1596-1597.

8. Arch. dép. d'Eure-et-Loir, registre d'état civil protestant d'Authon-du-Perche (non coté). Ces progrès dans l'enregistrement des BMS à partir de 1668 s'expliquent surtout par l'application du Code Louis.

9. FARCY, Jean-Claude et GARnOT, Benoît, Paroisses et communes de France, Eure-et-Loir, Paris, CNRS, 1990, p. 109.

10. Arch. dép. d'Eure-et-Loir, G 843, 1670, visite archidiaconale d'Authon. 
Évolution de la population réformée d'Authon au cours du XVII siècle

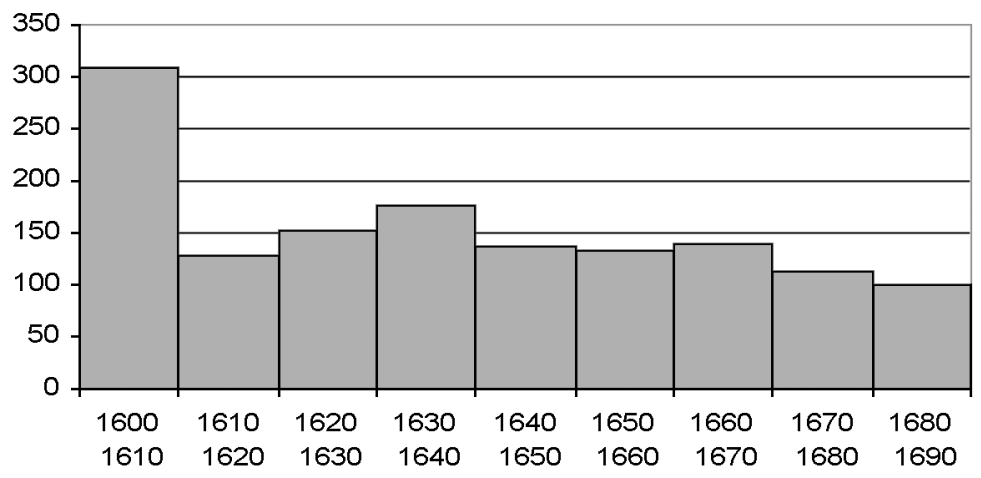

Le premier élément qu'il faut relever à la lecture de ce graphique, c'est l'importance de la population réformée entre 1600 et 1610, importance mise en valeur par la chute brutale qui lui succède. Cette forte diminution, dès les années 1610, semble liée aux caractéristiques de L'Église. En effet, en 1606, la communauté huguenote achète un bâtiment dans le bourg pour lui servir de lieu de culte ${ }^{11}$. Auparavant, les prêches se tenaient dans des maisons particulières ou dans un autre temple dont l'existence demeure inconnue. Toutefois, parallèlement, dès les premières années du pastorat de Jacques Couronné, certaines assemblées se déroulent à l'extérieur de la ville, comme le précise le ministre : « Registre et estat des mariages célébrez en l'église réformée d'Authon et quelquefois ailleurs selon les nécessités et circonstances des personnes et lieux où le pasteur d'icelle a esté appelé ${ }^{12}$. " Ainsi, jusqu'en 1607, 25 \% des assemblées ont lieu hors d'Authon. Pendant cette décennie, seule la moitié des huguenots réside effectivement à Authon, les autres vivant dans les paroisses des alentours, près de lieux de culte qu'on pourrait qualifier d'annexes. L'Église se distingue donc par un caractère de dispersion de ses lieux de culte comme de ses fidèles. Cette spécificité se retrouve dans d'autres Églises beauceronnes, telles Bazoches, Dangeau ou Marchenoir ${ }^{13}$. Or, si, à partir de 1607, la communauté se déplace beaucoup moins, l'aire géographique desservie par le pasteur se réduit et les protestants habitant loin ne viennent pas au temple à Authon. Ils se dirigent vers des Églises plus proches de leur domicile, Chartres, Dangeau, Châteaudun, Le Mans ou Bellême. C'est ainsi qu'on peut comprendre cette chute importante de population entre la décennie 1600 et la suivante. La communauté se replie alors sur elle-même, sur ses éléments forts, les

11. FARCY, Jean-Claude et GARNOT, Benoît, Paroisses et communes de France, Eure-et-Loir, op. cit., p. 144.

12. Arch. dép. d'Eure-et-Loir, registre d'état civil protestant, non coté.

13. Gueneau, Yves, Protestants du Centre, 1598-1685, thèse de troisième cycle, Université François Rabelais, Tours, 1982, p. 121. 
familles présentes dès 1600 dans le registre protestant. Il s'agit en particulier des dynasties Biard, Brunet, La Chassaigne, Desfontaines, Doisemont, Fouet, Gouin, Menou, Pezze, Renault, Robethon et Souchay.

Après cette baisse, la population s'équilibre durablement autour de cent quarante-cinq nouveaux individus par décennie. Les années 1620-1630 connaissent d'ailleurs un accroissement de la population. De nouvelles familles intègrent la communauté réformée. On compte parmi elles les Chappet, Des Rats, Rochay, Chedieu, Treizevent et Vannier. Ainsi, la population parvient à se perpétuer sans trop de difficultés : quelques hommes se marient et fondent une famille à Authon; leurs enfants adoptent la même démarche et ils compensent alors les pertes occasionnées par les familles composées de peu de membres et qui s'éteignent au cours du siècle, comme les Raintu, les Ridou, ou enfin les Froger, dont les cinq enfants sont des filles.

La stabilité démographique de la population huguenote s'explique également par la résistance que ses ressortissants entretiennent face aux accidents épidémiques et météorologiques rythmant le XVII ${ }^{\mathrm{e}}$ siècle. La mortalité des enfants, comme celle des adultes, est inférieure à la moyenne nationale - et catholique - et l'espérance de vie est en revanche supérieure à la moyenne avec cinquante-quatre ans et demi au vingtième anniversaire ${ }^{14}$. La communauté apparaît au premier abord stable et se distingue aussi par ses spécificités religieuses.

On trouve tout d'abord l'alphabétisation, caractéristique majeure des communautés protestantes : Calvin recommandait en effet la lecture de la Bible par chaque père de famille aux membres de son foyer. Ainsi, l'Église de Sancerre possède au XVII ${ }^{\mathrm{e}}$ siècle un taux d'alphabétisation de $70 \%$ et celle d'Aubusson de $83 \%{ }^{15}$. À Authon, il est de 35,3\% sur un siècle. Ce chiffre est un minimum car il est extrêmement difficile d'étudier les signatures avant 1628 : le ministre propose rarement aux témoins, parents et mariés de signer les actes de l'état civil. Alors, si l'on ne prend en compte que la population postérieure à 1630 et le nombre de signatures correspondant, on obtient un total de $52,3 \%$. On est encore loin des chiffres des riches villes du centre de la France mais on s'éloigne des pourcentages catholiques : ce résultat doit être plus proche de la vérité.

De multiples indices tendent à témoigner de la bonne culture religieuse des calvinistes de l'Église d'Authon : le choix et la variété des prénoms, l'af-

14. Lors de la peste de 1603, Jacques Couronné se trouve « contraint de rompre l'exercice public " après le culte du 21 septembre. Les fidèles quittent la paroisse et nombre d'entre eux se réfugient à Dangeau. À la date du 27 janvier 1604, à la fin de l'épidémie, les calvinistes constatent qu'aucun d'entre eux n'est décédé de la peste, alors que 59 catholiques en sont morts entre le 10 septembre et le 14 octobre. En revanche, la communauté subit la crise de la Fronde et celle de l'avènement. Pour cette dernière, le nombre de baptêmes passe de 11 en 1660 à 6 en 1661 et à 3 l'année suivante. Le nombre de mariages, de 3 à 4 les années normales, est de 0 en 1661 et 1662 et de 1 en 1663 .

15. Gueneau, Y., Les Protestants du Centre, op. cit., p. 279 bis. 
franchissement des prescriptions ancestrales liées aux temps clos catholiques d'abstinence, le délai entre la naissance et le baptême de l'enfant et la sérénité face à la mort évoquent l'apprentissage et l'acceptation de nouvelles conceptions de vie, synonymes d'une transformation solide des mœurs et d'une adhésion profonde à la nouvelle confession. Même si les registres du consistoire n'ont pas été conservés, aucun conflit majeur ne semble avoir secoué cette Église et ses pasteurs. Le premier des ministres connus, Jacques Couronné, a officié de décembre 1597 à mai 1644. Après le court pastorat d'Arbaud (juillet 1644-février 1646), René Rousseau lui succède entre avril 1646 et août 1664, puis Paul Joly (octobre 1664-octobre 1673), Marin Grossetête des Mahis (octobre 1673-avril 1676), Antoine Catel (juin 1676-juin 1679) et enfin Philippe Trouillard (juin 1679-avril 1684). Tous sont issus de milieux aisés. À l'exception de Couronné et de Rousseau, ils restent peu de temps à Authon : peut-être cherchent-ils à s'installer dans une autre Église aux ressources plus importantes et aux fidèles plus nombreux. C'est le cas du plus célèbre d'entre eux, Marin Grossetête des Mahis : né en 1649, après des études à Saumur, Genève et Oxford, il est nommé à Authon puis à Orléans. C'est dans cette ville qu'il abjure en 1683 et il publie à cette occasion La vérité de la religion catholique prouvée par l'Écriture Sainte et la Tradition; il devient alors chanoine de la cathédrale d'Orléans et meurt dans cette ville le 16 octobre $1694^{16}$.

Si les réformés semblent avoir intégré divers éléments d'appartenance au protestantisme, 77 \% des individus qui ne savent pas signer ont une profession inconnue et le pasteur n'a pas précisé cette donnée dans la moitié des cas. On peut donc supposer qu'une partie de ces individus correspond à des personnes modestes. Yves Guéneau précise d'ailleurs qu'il y a souvent " une répugnance à inscrire sur les registres d'état civil des professions, sans doute considérées comme trop humbles ${ }^{17}$ ". Ainsi, seule la moitié des protestants a une profession identifiée. Les résultats obtenus ne permettent donc d'apporter qu'une appréciation globale sur la société calviniste authonaise.

\section{Répartition socio-professionnelle des calvinistes à Authon}

\begin{tabular}{|c|c|c|c|c|c|}
\hline bourgeois & nobles & artisans & ruraux & total & \\
\hline effectif & 118 & 41 & 33 & 0 & 192 \\
pourcentage & 61,5 & 21,3 & 17,2 & 0 & 100 \\
\hline
\end{tabular}

Ainsi, aucun protestant ne semble travailler la terre. Tous les ruraux rencontrés à Authon entre 1668 et 1690 sont catholiques. Ils sont au nombre de quatre-vingt-neuf, soit $22,4 \%$ de l'effectif catholique. Ils ne sont donc pas majoritaires, Authon n'est pas une paroisse agricole. Cette absence de ruraux calvinistes se retrouve dans la province d'Anjou-Touraine-Maine,

16. HAAG, Émile et Eugène, La France protestante, Genève, Slatkine reprints, 1866.

17. Gueneau Y., Les Protestants du Centre, op. cit., p. 212. 
dans l'Église réformée du village de Saint-Aignan, le bourg de Bellême ou, plus compréhensible, l'Église du Mans ${ }^{18}$. En revanche à Dangeau, l'Église réformée de la province d'Orléanais-Berry la plus proche, $9 \%$ des calvinistes appartiennent à ce groupe ${ }^{19}$ : quatre sont vignerons et neuf sont laboureurs. En moyenne, 11 \% de la population réformée totale de cette province appartiendrait à cette catégorie socioprofessionnelle ${ }^{20}$. Or, pour Yves Guéneau, " ces chiffres ne doivent pas faire illusion "; selon lui, si en Nivernais, en Bas-Berry et en Marche, aucun rural n'était calviniste, en revanche, en Beauce, la majorité des protestants devait appartenir à ce groupe. Qu'en conclure? La communauté authonaise apparaît différente de ses voisines beauceronnes, sans que l'on puisse affirmer que la moitié des protestants dont la profession est inconnue ne travaillait pas la terre. Toutefois, aucun élément ne le laisse penser.

Les artisans non plus ne sont pas majoritaires dans la communauté calviniste. Ils forment moins d'un cinquième des hommes dont la profession est connue, alors qu'à Dangeau, ils représentent un tiers de l'effectif de l'Église ${ }^{21}$. Pourtant, Authon est l'un des grands centres de fabrication d'étamine de l'Ouest de la France : les étamines étaient envoyées à Tours, à Orléans et à Paris ${ }^{22}$. En fait, les artisans sont en grande partie catholiques : on en trouve deux cent vingt-deux entre 1668 et 1690 dans le registre d'état civil établi par le curé, soit 55,8 \% des catholiques. $66 \%$ des artisans réformés travaillent le textile et autant d'artisans catholiques font de même : le tissage est bien l'activité principale de la paroisse. Les autres sont artisans du vêtement, et notamment gens d'aiguille, profession fréquemment sensible à la Religion Réformée ${ }^{23}$. En revanche, dans les autres activités, artisanat de luxe, du cuir, de l'habitation, de l'outillage et de l'alimentation, les catholiques sont plus nombreux. On retrouve également cette faiblesse, voire cette absence, à Châteaudun et à Dangeau.

De son côté, la noblesse structure la communauté réformée : les nobles sont présents aux assemblées, ils parrainent, ils sont anciens, ils offrent des lieux pour le culte - lorsque celui-ci ne se déroule pas au temple - et ainsi, non seulement maintiennent les effectifs, mais les retiennent par leur seule présence. D'après Jean-Marie Constant, la charge d'ancien, de délégué au synode, donne aux nobles un pouvoir local que le catholicisme ne pourrait leur apporter ${ }^{24}$. C'est cette fonction qui explique en partie leur importance

18. Pour les Églises de Saint-Aignan et du Mans : Arch. dép. de la Sarthe, 111 AC 997 (registres protestants). Pour Bellême : MENARD, Céline, La Communauté protestante de Bellême au XVII siècle, mémoire de maîtrise, Université du Maine, 2004, p. 56.

19. Gueneau, Y., Les Protestants du Centre, op. cit., p. 144.

20. Ibidem, p. 212.

21. Ibid., p. 197.

22. BoISSIERE, Jean et MichAUD, Claude, L'Intendance d'Orléans à la fin du XVIre siècle. Édition critique du mémoire "Pour l'instruction du duc de Bourgogne », Paris, CTHS, 1989, p. 127.

23. Gueneau, Y., Les Protestants du Centre, op. cit., p. 198.

24. Constant, Jean-Marie, Nobles et paysans en Beauce aux XVI et XVI siècle, thèse de $3^{\text {e }}$ cycle, 1978, Université de Lille III, service de reproduction des thèses, 1981, p. 340. 
dans les Églises réformées : ils représentent 21 \% de la communauté. Ce taux est important si l'on s'en tient au pourcentage des nobles généralement admis par rapport au total de la population française : 1 à 1,5 \% de la population était noble sous l'Ancien Régime ${ }^{25}$. En revanche, aucun noble n'appartient à la communauté catholique. Cette proportion de familles nobles se retrouve aussi dans d'autres communautés de Beauce, Dangeau, avec 23,5 \% de calvinistes nobles, ou Bazoches-en-Dunois, $15 \%{ }^{26}$. La noblesse a donc véritablement une place essentielle dans les Églises de Beauce, par le nombre de ses représentants, mais aussi, et surtout, par son rôle.

Enfin, avec 61 \% des hommes dont la profession est connue, la bourgeoisie est le principal groupe social de la communauté réformée.

Répartition des bourgeois selon leur activité

\begin{tabular}{|c|c|c|c|c|c|c|c|}
\hline & marchands & sieurs & $\begin{array}{c}\text { hommes } \\
\text { de loi }\end{array}$ & $\begin{array}{c}\text { corps de } \\
\text { santé }\end{array}$ & lettrés & militaires & total \\
\hline $\begin{array}{c}\text { effectif } \\
\%\end{array}$ & 41 & 27 & 25 & 12 & 7 & 6 & 118 \\
\hline
\end{tabular}

On trouve principalement des hommes de loi et des professions de la santé, bien représentés comme dans toutes les communautés du centre de la France. Dans la province d'Orléanais-Berry voisine, seules les communautés de Gien et Issoudun dépassent en nombre les chiffres trouvés pour Authon ${ }^{27}$. Les hommes de loi authonais se composent d'un bailli, trois notaires, trois procureurs, cinq huissiers et treize avocats. De nombreux juristes protestants choisissent en effet le métier d'avocat car il s'exerce librement : il n'y a pas besoin de lettre de provision ${ }^{28}$ et, de ce fait, son activité peut difficilement être entravée. De même, on trouve à Authon trois apothicaires, quatre chirurgiens et cinq médecins calvinistes. Selon Robert Muchembled, « les médecins étaient rarement installés à la campagne avant le XVIII ${ }^{\mathrm{e}}$ siècle ${ }^{29}$ " et, en effet, aucun catholique n'exerce cette profession. Mais ils sont présents au XVII ${ }^{\mathrm{e}}$ siècle dans la communauté calviniste, à Authon comme à Bellême ${ }^{30}$. Quatre des cinq médecins appartiennent à la puissante famille Robethon, celle qui peut-être instaura le protestantisme à Authon et façonna l'Église réformée.

Parmi les groupes sociaux numériquement les plus importants, on remarque aussi les marchands. Yves Guéneau précise que pour la province d'Orléanais-Berry, " la catégorie marchande est la plus nombreuse ${ }^{31}$ ". Elle représente 85 \% des bourgeois à Aubusson, 62,7 \% à Châteaudun, 44 \% à

25. NASSIET, Michel, La France du second XVIr siècle, 1661-1715, Paris, Belin Sup., 1997, p. 58.

26. Gueneau, Y., Les protestants du Centre, op. cit., p. 218.

27. Ibidem, p. 173 ter.

28. LIGOU, Daniel, Le Protestantisme en France de 1598 à 1715, Paris, SEDES, 1968, p. 204.

29. MuchEMBLED, Robert, Société, cultures et mentalités dans la France moderne, XVI -XVIII siècle, Paris, 2001, p. 56.

30. Menard, C., La Communauté protestante de Bellême, op. cit., p. 53.

31. Gueneau, Y. , Les Protestants du Centre, op. cit., p. 173. 
Blois et $12 \%$ à Dangeau ${ }^{32}$. Les chiffres apparaissent relativement disparates. À Authon, les marchands semblent au premier abord beaucoup moins nombreux que dans d'autres communautés calvinistes, mais il faut préciser qu'Authon n'est qu'une bourgade du Perche, où le négoce n'a pas le même rôle économique que dans les villes du Val de Loire. Les marchands sont cependant la catégorie sociale la plus importante de l'Église. Certains indices amènent à penser qu'il existe deux catégories de marchands : plusieurs tisserands vendent leurs productions, et le passage de la fabrication au négoce apparaît relativement facile. Mais la plupart sont de véritables négociants. Pour Jean-Marie Constant, le marchand est en Beauce « l'armature sociologique des bourgades où il vit ${ }^{33}$ ». Il déplace beaucoup d'argent : il achète, vend et s'enrichit. Dix d'entre eux peuvent même s'offrir une seigneurie. C'est le cas de François Biard, marchand et sieur de la Marette. Le marchand vit généralement au-dessus de sa boutique, ellemême située près des halles d'Authon. De son côté, Paul Desfontaines possède " une petite maison [...] couvers de tuile et bardereau contenant chambre basse à feu, grenier dessus, un bas costé sur la rue et une estable au bout de ladite chambre [...] Item ledit jardin joignant à ladite chambre et estable ${ }^{34}$ ". Cette image du logis rappelle celle des marchands d'Oizé, au sud du Mans, étudiés par Anne Fillon : "Les marchands habitent des maisons comprenant généralement deux pièces - une chauffante et une froide avec plus ou moins de dépendances selon leurs activités ${ }^{35}$. "La chambre chauffée est la pièce à vivre, la salle froide destinée aux réserves. François Biard possède un niveau de vie confortable, il dispose d'un mobilier important, notamment tables et armoires, signe de richesse et d'ouverture à la nouveauté ${ }^{36}$. Mais sa demeure n'a pas la notion de confort qu'on trouve chez d'autres bourgeois d'Authon.

Les membres de la famille Robethon représentent en effet le niveau supérieur de la bourgeoisie : ils possèdent des terres et vivent noblement. Ainsi, la demeure de Paul Robethon est " un logis manable en forme de pavillon se constituant en deux chambres basses, une petite cuisine et garderobbe, deux chambres haultes et garderobbe et un grenier sur tout ledit logis avec une cave voultée soubs la moytié de selluy et au bout une escurie, et derrière [...] une court ${ }^{37}$ " : les pièces apparaissent nombreuses et chacune a son utilité. Chez Jacques Robethon, on trouve des chandeliers, des miroirs, des tapis, de l'argenterie; le bois des meubles est plus beau,

\section{Ibidem.}

33. Constant, Jean-Marie, Nobles et paysans en Beauce, op. cit., p. 90.

34. Arch. dép. d'Eure-et-Loir, H 2584, minutes notariales de la seigneurie du prieuré à Authon, 15 octobre 1642, vente.

35. FILLON, Anne, Fruits d'écritoire, Le Mans, Laboratoire d'histoire anthropologique du Mans, 2000, p. 79.

36. Arch. dép. d'Eure-et-Loir, E 4322, minutes notariales d'Henri Chedieu, 6 et 8 mai 1673, inventaire après décès.

37. Arch. dép. d'Eure-et-Loir, H 2585, minutes notariales de la seigneurie du prieuré à Authon, 16 novembre 1620, reconnaissance de cens. 
plus riche, les couvertures sont en laine blanche et verte, la cuisine regorge d'ustensiles ${ }^{38}$. La famille Robethon semble par conséquent appartenir à l'élite de cette communauté. Ses membres sont avocats ou médecins, mais ce ne sont pas leur profession qui leur permet de vivre. Ils sont avant tout propriétaires de terres qu'ils font exploiter et vivent dans des demeures où le confort est apparu.

La communauté réformée apparaît donc véritablement et prioritairement bourgeoise. La population y est majoritairement aisée puisque seuls $20 \%$ des notables authonais appartiennent à la confession catholique. Toutefois, la présence importante des nobles à Authon est originale : dans les Églises beauceronnes telles Bazoches-en-Dunois, Lorges ou Marchenoir, la noblesse est associée à une forte communauté d'artisans ${ }^{39}$. Ainsi, seuls Authon et Dangeau sont caractérisés par une population majoritairement bourgeoise, puis noble, et ayant une minorité d'artisans.

La fortune de l'Église semble d'autre part correspondre aux caractéristiques sociales de la communauté. Elle est connue par les actes synodaux ainsi que par le rattachement des biens du consistoire à la fabrique paroissiale d'Authon après la Révocation. En 1649, l'Église fait don de 20 livres au synode et de 51 livres aux académies ${ }^{40}$. En 1679 , elle verse 30 livres et n'en reçoit rien ${ }^{41}$. Selon Henri Lehr, il est rare que les Églises du nord de la France s'en sortent financièrement sans l'aide du synode, comme cela semble être le cas d'Authon. Ainsi, l'Église d'Herly, en Picardie, " sans être dans la misère, coûte plus cher au synode qu'elle ne lui rapporte ${ }^{42}$ ". Si les grandes Églises de Picardie, Calais et Saint-Quentin, octroient respectivement 440 et 220 livres au synode, la plupart des Églises picardes donnent entre 40 et 50 livres. Ainsi, Authon paraît avoir des finances semblables à celles de la moyenne des Églises de Picardie.

Par ailleurs, lors de la remise des biens du consistoire à la fabrique, en 1687, les possessions d'Authon comprenaient un capital de 2719 livres en numéraire et plusieurs rentes foncières, dont une de 25 livres et une autre de 5 livres ${ }^{43}$. Yves Guéneau a comparé les biens de plusieurs consistoires, estimés après la Révocation ${ }^{44}$. Il a mis notamment en parallèle les Églises de Sancerre, Orléans, Blois et Marchenoir. Les capitaux et les rentes sont connus pour ces quatre communautés, ce qui permet de situer Authon par

38. Arch. dép. d'Eure-et-Loir, E 4325, minutes notariales d'Henri Chedieu, 24 septembre 1681 , inventaire après décès.

39. BoISSON, Didier et LIPPOLD, Christian, « Les protestants du centre de la France et du Bassin Parisien et la révocation de l'édit de Nantes ", Bulletin de la Société de l'Histoire du Protestantisme Français, 2002, p. 349.

40. LEHR, H., La Réforme et les églises réformées, op. cit., p. 321.

41. Ibidem, p. 323.

42. TANTIN, Valérie, Étude de la communauté protestante d'Herly avant et après la révocation de l'édit de Nantes : les relations entre calvinistes et catholiques de Picardie, mémoire de maîtrise, Université Paris IV-Sorbonne, 1993, p. 70.

43. Guillon, François, " Le protestantisme à Authon... ", op. cit., p. 155.

44. Gueneau, Yves, Les Protestants du Centre, op. cit., p. 350 bis. 
rapport à ces Églises. La plus riche d'entre elles est celle d'Orléans, avec au moins 2580 livres de capital et deux rentes lui rapportant 528 livres par an. Le capital d'Authon est alors supérieur à celui d'Orléans de 140 livres et si ses rentes lui rapportent a priori moins, elles sont plus nombreuses. Authon apparaît donc comme une Église aux revenus moyens comparativement à la Picardie, mais elle appartient aux Églises riches face aux communautés de la Loire.

Par conséquent, les réformés sont minoritaires dans le bourg, mais ils compensent leur faiblesse numérique par leur poids social. Quelles sont alors les relations entre les individus des deux confessions et comment se manifestent-elles?

\section{Un équilibre trouvé avec les catholiques}

Il est possible de supposer, à la suite d'Elisabeth Labrousse, " que la coexistence pacifique était plus facile dans les bourgs dans lesquels les deux confessions reconnues en France se trouvaient dans un certain équilibre, parce qu'il semble probable que là où l'une était fortement majoritaire, elle cédait aisément à la tentation d'être oppressive ${ }^{45}$ ". Divers éléments nous permettent de découvrir les relations entre les deux communautés religieuses.

Tout d'abord, il faut bien voir que pratiquement toutes les grandes familles réformées ont aussi des membres catholiques. Seule la lignée des Menou n'apparaît jamais dans le registre catholique avant 1690. Ainsi, trente-neuf hommes appartenant aux La Chassaigne se marient et ont des enfants à Authon au XVII ${ }^{\mathrm{e}}$ siècle et parmi eux, cinq sont protestants. La famille est donc partagée entre les deux confessions. Elisabeth Labrousse indique effectivement que " la plupart des huguenots avaient une parenté demeurée catholique " et qu'il est " patent que le huguenot moyen se rendait au baptême et surtout au mariage et à l'enterrement de ses parents catholiques les plus proches ", malgré la Discipline qui interdit de telles rencontre et les risques de sanctions que le consistoire pouvait prendre ${ }^{46}$.

Les échanges professionnels montrent également que les habitants du bourg vivent en bonne entente : le négoce, les locations et les ventes, les prêts et les remboursements n'ont pas de frontières confessionnelles. Par ailleurs, il a déjà été évoqué le fait qu'aucun réformé ne pratique un métier de bouche : les boulangers, les bouchers et les marchands celliers sont tous catholiques. Les artisans de l'équipement, de l'habitation et de l'outillage, les charpentiers, les couvreurs, les maréchaux et les charrons sont également catholiques. Dans la deuxième moitié du XVII ${ }^{\mathrm{e}}$ siècle, le fermier et le fournier du four banal, Jacques Rayer et Pierre Dupéage, sont aussi tous deux catholiques. Enfin, les hôtes de la Borde, de la Botte et

45. Labrousse, Élisabeth, La Révocation de l'Édit de Nantes. Une foi, une loi, un roi ?, Paris, Petite Bibliothèque Payot, 1990, p. 70-71.

46. Ibidem, p. 68. 
de la Croix-blanche appartiennent aussi à cette confession. Ainsi, dans sa vie quotidienne, le calviniste a souvent besoin de commercer avec un catholique.

Sur le plan religieux, les relations semblent également se dérouler sur ces mêmes bases. En premier lieu, six mariages mixtes, alliant catholiques et protestants, ont été célébrés. Ces mariages, désapprouvés par les autorités religieuses des deux confessions, sont tolérés au XVII ${ }^{\mathrm{e}}$ siècle par les deux communautés : le curé et le pasteur ne peuvent les refuser sous peine de voir leurs fidèles se tourner vers l'autre ministre. Chez les calvinistes, " quand un mariage bigarré avait eu lieu, après une pénitence publique du conjoint et de ses parents, le dimanche, au temple, le délinquant (ou la délinquante) était réconcilié et on n'en parlait plus ${ }^{47}$ ". Les anciens, comme le pasteur et le curé, sont des hommes de leur siècle, pour qui les impératifs familiaux peuvent suppléer les prescriptions religieuses. Les couples se marient dans le cadre de la religion de l'épouse. Pourtant, chaque conjoint conserve sa foi d'origine, même si le pasteur ou le curé n'accepte pas cette situation. Ainsi, le 15 août 1638 a lieu le baptême de "Jan, fils de Julian Guy, qui vit encore en superstition de l’Église romaine, dont Dieu le veuille retirer ${ }^{48}$ ". Pour quatre des six mariages, les garçons nés de cette union sont baptisés dans la religion de leur père et les filles dans celle de leur mère. Seul Julien Guy, comme on vient de le voir, cédant peut-être à sa femme et au pasteur ou indifférent aux questions religieuses, baptise ses deux enfants, Marie et Jean, au temple de l'Église réformée d'Authon. En revanche, Ambroise Pezze et Madeleine Germond alternent entre baptêmes selon l'ordonnance catholique et baptêmes protestants : Gervais, né en 1598, Madeleine, née en 1599, Marie, née en 1606, Antoine, né en 1613 et Ambroise, né en 1617, sont baptisés à l'église, alors que Paul, né en 1609, Jacques, né en 1611 et Jean, né en 1614 , sont baptisés au temple. Aucun ordre ne semble pouvoir se dégager de leurs choix. Toutefois, l'éducation maternelle prévalant et influençant souvent les enfants, à l'âge adulte, Jacques Pezze, bien que baptisé protestant, choisit la religion catholique.

Plus curieusement, trois petits calvinistes naissent au sein de la communauté authonaise en 1671 et sont baptisés dans l'église paroissiale par le ministre de la religion réformée " pour cause de faiblesse " des enfants. Le temple est en effet à cette époque éloigné du bourg et le déplacement des enfants pourrait leur être fatal. Parmi ces trois enfants se trouve Catherine Joly, la propre fille du ministre réformé. Les relations entre le curé et le pasteur doivent donc être extrêmement bonnes pour que le premier consente à ouvrir son église et que le second accepte d'y entrer. L'importance pour le prêtre catholique de sauver des âmes et l'espoir de voir ces familles se convertir peut éventuellement expliquer son geste. On retrouve un événement identique à Bellême : en 1651, le curé de Saint-

47. Ibid., p. 69.

48. Arch. dép. d'Eure-et-Loir, registre d'état civil protestant d'Authon, non coté. 
Sauveur ouvre les portes de son Église, de nuit, afin que ses fidèles « ne voient pas ce triste spectacle ${ }^{49}$ ".

Enfin, sur le plan géographique, on s'aperçoit que les deux communautés ont, consciemment ou inconsciemment, organisé le bourg afin de limiter les tensions, voire les incidents. Tout d'abord, six actes notariés ${ }^{50}$ et deux actes de justice ${ }^{51}$ font mention de l'adresse de dix protestants au cours du siècle. Le chiffre est extrêmement faible, presque inutilisable, sauf à remarquer que cinq d'entre eux vivent dans la même rue et trois autres dans les rues adjacentes. Cette rue, où demeurent Jacques Robethon, Paul Desfontaines, François Biard, Théodore Treizevent et Abraham Menou, est la rue Basse, au sud du bourg, près des fossés, où est établi le temple de la communauté. On trouve également tout près les demeures de Marin Gardon, Jean Guelot et Daniel Renau. D'autre part, lorsque le notaire ne précise pas l'adresse exacte des individus dont il parle, il a l'habitude d'évoquer leurs voisins. Ainsi, en 1655, lorsque François Biard loue un jardin à Louis Souchay, sieur des Fossés, bourgeois protestant, il indique que le jardin est " situé en cedit lieu d'Authon, joignant de deux pars audit sieur des Fossés, d'autre part à maistre Jacques Chedieu ", avocat huguenot. Ces indications accentuent l'impression de regroupement des calvinistes au sud du bourg, près de leur temple, voisins les uns des autres, formant alors une entité géographique solide face à la majorité catholique, elle-même installée au nord, près de l'église paroissiale Saint-André. Les halles séparaient alors les deux territoires.

En revanche, les Authonais ont gardé un cimetière commun, le cimetière paroissial, situé près de l'église. Il est simplement séparé en deux parties, une pour chaque confession, afin que chacun puisse être enterré en terre chrétienne. Pour les Authonais, les différences entre catholicisme et calvinisme ne semblent pas si fondamentales et les protestants ne sont pas perçus comme des hérétiques que l'on doit exclure de la terre sacrée. Le partage a pourtant eu l'originalité de laisser " la croix du cimetière [...] du costé occupé par les hérétiques ${ }^{52}$ ". Le sacristain, de son côté, inhume tous les morts, quelle que soit leur confession. Selon Jean Quéniart, cette conduite est dictée par des impératifs économiques ${ }^{53}$. Toutefois, cette décision suppose que le fossoyeur catholique ait la conviction d'agir conformément à ses croyances religieuses.

Il en est de même à l'école : l'instruction des catholiques comme des protestants se fait dans l'école paroissiale. L'article XxII de l'édit de Nantes

49. Arch. dép. de l'Orne, 3 E 495/31, cité par MenaRd, Céline, La Communauté protestante de Bellême, op. cit., p. 79.

50. Arch. dép. d'Eure-et-Loir, H 2580, H 2584, H 2585, minutes notariales concernant la seigneurie du prieuré d'Authon, 1620, 1623, 1630, 1642, 1655, ventes, baux et reconnaissances de cens; E 4325, minutes notariales Henri Chedieu, 1680, bail.

51. Arch. dép. d'Eure-et-Loir, B 2865, registre de plaintes, 1690, plaintes contre Abraham Menou et Jacques Taunay.

52. Arch. dép. d'Eure-et-Loir, G 820, registre de la visite archidiaconale du Dunois, 1630.

53. QuenIART, Jean, La Révocation de l'édit de Nantes, Paris, Desclée de Brouwer, 1985, p. 71. 
décrète en effet qu'il ne doit y être " fait différence ni distinction pour le regard de ladite religion à recevoir les écoliers pour être instruits ès [...] écoles ". Un acte de catholicité n'est donc pas nécessaire et les enfants protestants peuvent y être admis. Cependant, les divers maîtres d'école qu'Authon a connus au XVII ${ }^{\mathrm{e}}$ siècle sont catholiques. Or, le but de l'école n'est pas seulement d'apprendre aux enfants à lire, écrire et compter, " les petites écoles transmettent en effet la religion et la morale dominantes, modelant des esprits dociles et des cœurs chrétiens, avant d'éveiller les intelligences ${ }^{54}$ ». Pourquoi alors, dans ces conditions, les parents réformés envoient-ils leurs enfants à l'école paroissiale? C'est l'archidiacre du Dunois qui, au cours d'une de ses visites, en 1678, donne la réponse : il signale que le maître d'école instruit les enfants des huguenots selon leur religion, qu'il leur apprend " à chanter les psaumes de David suivant la version de $\operatorname{Marot}^{55}$ ". Ainsi, le maître d'école catholique ne cherche pas à convertir les enfants, il semble respecter la foi de chacun, comme le fait aussi celui du bourg beauceron de Patay qui, en 1670, " enseigne les enfants des huguenots et même leur fait lire des livres de leur religion " ou un autre qui, en 1678, comme à Authon, apprend " auxdits huguenots à chanter les psaumes de David suivant la version de $\operatorname{Marot}^{56}$ ".

Les Authonais sont avant tout des parents, des amis, des voisins, des collègues et la religion de chacun est moins fondamentale que les liens villageois, primordiaux pour ces hommes de l'Ancien Régime. Robert Sauzet parle « d'une véritable symbiose entre catholiques et réformés ${ }^{57}$ ». Lorsque l'Église réformée est solidaire et forte, le clergé catholique et ses fidèles entretiennent de bons rapports avec elle. C'est ainsi que ni Blaise Féron ni Nicolas Le Maire, archidiacres successifs du Dunois, ne reçoivent de la part des habitants, au cours de leurs visites, de doléances contre les protestants. En fait, quand les tensions apparaissent entre catholiques et huguenots authonais, ce ne sont généralement pas les populations qui en sont responsables.

\section{Une communauté sur le repli en fin de siècle}

C'est le clergé catholique qui, dans la seconde moitié du XvII ${ }^{\mathrm{e}}$ siècle, inquiète la communauté protestante. Plus précisément, si le curé de la paroisse ne semble pas si hostile aux réformés, son supérieur, l'archidiacre du Dunois, le place face à ses devoirs et rappelle la " noirceur de l'héré$\operatorname{sie}^{58}$ " aux catholiques du bourg d'autant plus tentés de l'oublier qu'ils

54. Benoît GaRnot, Les Villes en France aux XVI', XVII et XVII' siècles, Paris, Ophrys, 1989, p. 94.

55. Arch. dép. d'Eure-et-Loir, G 844, registre de la visite archidiaconale du Dunois, effectuée à Authon le 16 octobre 1678.

56. Robert SAUZET, « Les procès-verbaux des visites pastorales du diocèse de Chartres au XVII e siècle ", Archives de Sociologie des Religions, tome 35, 1972.

57. Ibidem, p. 54.

58. Labrousse, E., La Révocation de l'édit de Nantes, op. cit., p. 50. 
vivent près des huguenots. Le clergé est souvent "l'élément déclencheur ", nous dit Jean Quéniart ${ }^{59}$. Ainsi, c'est l'archidiacre, venu en visite dans la paroisse d'Authon, qui inscrit et transmet à l'évêque de Chartres tous les éléments de coexistence pacifique entre catholiques et huguenots qui nous sont parvenus : école, marguillier et cimetière commun. Par des menaces, il a pu les faire interdire ou supprimer.

En fait, les divers événements affectant l'Église réformée ont toujours pour cause la volonté catholique de réduire à néant les protestants et leur organisation. Ainsi, au mois d'août 1664, le pasteur René Rousseau quitte les calvinistes et il est remplacé au mois d'octobre par Paul Joly ${ }^{60}$. Dans cet intervalle, pendant lequel la communauté se trouve fragilisée, une demande sur la légitimité du temple d'Authon est transmise par un membre du clergé aux commissaires chargés de vérifier le respect de l'édit de Nantes dans les provinces du royaume ${ }^{61}$. Très rapidement, le Grand Conseil " interdit l'exercice de la religion réformée dans la ville d'Authon ${ }^{62}$ ". Le temple doit donc être détruit car l'exercice du culte est prohibé intra-muros. Dans ces mêmes années, les Églises de Mer et d'Aubusson ont subi une condamnation identique et ont reçu l'ordre de s'exiler hors du centre de la ville $^{63}$. Le but de cette interdiction est d'éloigner le temple, et donc les chants et les cérémonies, de la vue des fidèles catholiques. Mais la suppression d'un temple n'entraîne pas la conversion de la population protestante. La communauté ne construit pas non plus un nouveau temple dans les faubourgs comme à Mer ou à Aubusson. Ainsi les réformés émigrent à quelques kilomètres d'Authon, au château de la Chauverie, situé sur la paroisse de Luigny, dont le seigneur est protestant. La pérégrination de l'Église calviniste authonaise reprend selon une tradition mise en place au début du $\mathrm{XVII}^{\mathrm{e}}$ siècle et dans des lieux déjà connus par les deux générations précédentes. Peu après, le prêche se rapproche d'Authon et s'installe dans la demeure seigneuriale de Champs, paroisse de Melleray, appartenant à un autre noble de la communauté réformée d'Authon. Le culte y a lieu de 1665 à 1672. Toutefois, dès 1670, l'archidiacre s'insurge contre l'existence d'un prêche calviniste sur la paroisse de Melleray : "Monsieur de Champs, de la RPR, a establi un presche dans sa maison où luy, ses domestiques et même les hérétiques du voisinage s'assemblent et on y fait des prières et le presche ${ }^{64}$. " L'année suivante, il proteste à nouveau : " Nous

59. QUENIART, J., La Révocation de l'édit de Nantes, op. cit., p. 72.

60. Arch. dép. d'Eure-et-Loir, registre d'état civil protestant d'Authon, non coté.

61. Par la déclaration royale du 18 juillet 1656, qui ne connaît un début d'application qu'en avril 1661, à la simple demande du clergé, deux commissaires (un catholique, l'intendant, et un protestant, un magistrat ou un gentilhomme) sont envoyés sur un lieu de culte et sont chargés de faire appliquer "à la rigueur " l'édit de Nantes.

62. GullLon, F., "Le protestantisme à Authon du Perche... ", op. cit., p. 148.

63. Borsson, Didier, Les Protestants de l'ancien colloque du Berry de la révocation de l'Édit de Nantes à la fin de l'Ancien Régime (1679-1789), ou l'inégale résistance de minorités religieuses, Paris, Honoré Champion, 2000, p. 106.

64. Arch. dép. d'Eure-et-Loir, G 843, visite archidiaconale de Melleray, 1670. 
avons appris que le sieur de Champs [...] continue de tenir des assemblées de la même sorte dans la maison ${ }^{65}$. " Il a ainsi incité la communauté à quitter Champs. Les réformés se dirigent alors vers un manoir proche d'Authon, Coutermay, appartenant à Louis Souchay, sieur de Gandelion, officier du duc d'Orléans et ancien de l'Église. Les calvinistes s'y retrouvent alors jusqu'à la disparition de leur Église.

Les tourments de la communauté reprennent en 1681, lorsque le clergé catholique entame une nouvelle procédure contre elle. Sont faites " inhibitions et défenses au sieur de Gandillon [Gandelion], aux ministres et anciens du consistoire et aux habitants du lieu d'Authon, de faire à l'avenir aucun exercice de leur religion au lieu de Coutermé, à peine de cinq cent livres d'amendes ${ }^{66}$ ". Les réformés en sont informés le 11 juillet 1681. Ils refusent de se soumettre, résistent en s'opposant à cette décision devant les commissaires de l'édit de la généralité d'Orléans. L'affaire est ensuite mise en appel devant le Conseil du Roi. Le texte du jugement n'a pas été conservé et, pourtant, la décision du Conseil du Roi est connue par la mention portée au bas des pièces de procédure, très explicite : « jugé le 13 mars 1684 - interdit ". L'Église réformée d'Authon est proscrite durant trois ans après la demande soumise par le clergé, preuve peut-être des hésitations du pouvoir alors que l'Église catholique ne connaît pas de telles réticences. Le dernier pasteur, Philippe Trouillard, termine ainsi le registre d'état civil :

«Le troisiesme jour d'avril 1684, Suzanne Taunay, fille de Jacques Taunay, maistre étaminier, et de Anne Brunet, sa femme, a esté baptisée par moy soussigné, cy devant ministre de ceux de la religion d'Authon et lieux circonvoisins qui s'assembloient à Coustermay, proche dudit Authon, m'estant transporté exprès sur les lieux, à la requeste du père de l'enfant, lequel m'est venu prié de ce faire lorsque je me retirois du pays pour obéir à un arrest du Conseil du Roi, lequel m'avoit esté signifié, portant injonction aux ministres des églises interdites de ne pas demeurer plus près que de six lieues des dites esglises et l'arrest d'interdiction de celle qui se recueilloit à Coustermay ne m'ayant esté signifié que lundy dernier, premier jour du dit, sur les deux ou trois heures de l'après midi; le père dudit enfant m'ayant trouvé à la terre nommée la Reyne Bouvière, près de Montmirail, distante de trois ou quatre lieues d'Authon, m'y estant arresté à cause de la feste de Pasques, dans le dessein de me retirer plus loin, le plus tost que faire se pourroit; ce que j'ay fait ayant eu permission du sieur lieutenant et procureur fiscal de la baronnie dudit Authon de baptiser ledit enfant, né aujourd'huy sur les trois heures du matin. "

Toutefois, entre juillet 1681 et mars 1684, l'exercice du culte calviniste se poursuit légalement. Précédemment, plusieurs autres lieux de culte proches ont eux aussi été interdits, La Ferté-Vidame en 1681, Châteaudun en 1682, Dangeau en 1683 et Bellême en janvier 1684; et au mois de

65. Arch. dép. d'Eure-et-Loir, G 844, visite archidiaconale de Melleray, 18 septembre 1671.

66. Archives nationales, série TT - Coutermé, partage d'avis des commissaires sur le droit d'exercice de la RPR - 1681, cité par François Guillon, "Le protestantisme à Authon..." op. cit., p. 154. 
mars 1684, c'est celui d'Authon. Avant cette date, plusieurs éléments témoignent cependant des difficultés rencontrées par les calvinistes dans leur vie quotidienne.

Le premier élément est démographique. À la fin du siècle, un phénomène nouveau apparaît, différent des crises classiques : on observe la diminution de la population protestante à partir de la décennie 1670. La chute s'accentue après 1680 et toutes les classes d'âge sont atteintes. Ce comportement nouveau de la communauté calviniste authonaise s'apparente à une crise prolongée, débutant peut-être dès 1673. Cette particularité se retrouve également dans de nombreuses communautés du Centre étudiées par Yves Guéneau, comme Châteaudun et Dangeau ${ }^{67}$. Elle peut s'expliquer par les conséquences de la politique royale : les persécutions subies par les huguenots entraînent abjurations et exils, et ceux qui restent attendent des jours meilleurs pour prendre un nouveau départ. Ainsi, entre 1678 et 1681, les décès des réformés authonais sont supérieurs aux baptêmes enregistrés par la communauté.

D'autre part, certaines professions contre les hommes de loi et le corps de santé sont mises en péril par la législation, appliquée de plus en plus strictement à l'approche de la Révocation. En effet, le 21 juillet 1664, le Conseil Privé décide d'annuler rétrospectivement toutes les lettres de mâ̂trise où il n'est pas mentionné que le titulaire est de religion catholique, apostolique et romaine. Les concurrents catholiques ont su veiller à l'exécution de ces arrêts qui les favorisaient. Ainsi, aucun protestant authonais ne devient homme de loi après 1670 et cinq chirurgiens sont enregistrés dans le registre catholique entre 1681 et 1698. Les détenteurs de ces arts paraissent donc choisis, à la fin du siècle, parmi les catholiques. Selon Élisabeth Labrousse, les calvinistes sont peu à peu exclus " de tous les postes administratifs et judiciaires, de tous les offices et de toutes les charges (notaires, procureurs, huissiers, sergents, etc.) ${ }^{68}$ ". Ce phénomène se confirme à Authon, comme partout ailleurs.

Par ailleurs, le parrainage élitiste augmente dans les dernières années, précédant l'interdiction de l'Église et la Révocation de l'édit de Nantes. Sur un siècle, 10,4 \% des parrains et marraines sont choisis pour leur appartenance à l'élite de la communauté. Mais, lors de la décennie 1670, 16,3\% des parrains appartiennent à la noblesse et entre 1680 et 1684, ce sont $38 \%$ des parrains qui sont nobles : l'accroissement des difficultés étant de plus en plus perceptible, les calvinistes veulent se prémunir. Les familles nobles sont alors très largement sollicitées. Ainsi, ce sont les nobles qui encadrent et soutiennent l'Église dans ses vingt dernières années. Ils prêtent leurs demeures pour que le culte ait lieu et résistent au clergé local. Ils sont actifs au niveau des institutions et présents aux cérémonies, en tant que parti-

67. Gueneau, Y., Les Protestants du Centre, op. cit., p. 234 et 243 à 245. Voir aussi BenEDICT, Philip, "La population réformée française de 1600 à 1685 ", Annales ESC, 1987, n 6, p. $1433-1465$.

68. Labrousse, E., La Révocation de l'édit de Nantes, op. cit., p. 155. 
cipants mais aussi comme témoins. Yves Guéneau indique d'ailleurs pour la région dont fait partie Authon : "Châteaudun mis à part, les lieux de culte ne se maintinrent en Beauce que là où les nobles restèrent fidèles à la Réforme $^{69}$ ", même si certains d'entre eux se convertissent au catholicisme.

En effet, les abjurations constituent la dernière source de tensions qui affaiblissent la communauté réformée. Les récits de deux conversions, effectuées au moment de la mort du converti, nous sont parvenus par l'intermédiaire des visites pastorales. Il s'agit de celle de Louis de Gallot, écuyer, sieur de Tilly, et de Timothée Le Hayer, écuyer, sieur de Bréval. Le premier est enterré le 3 mars 1670 dans le cimetière paroissial d'Authon, et le second est décédé en 1671 - la date précise de sa mort est inconnue. Le 19 septembre 1671, l'archidiacre du Dunois rapporte les propos du curé d'Authon, qui " auroit confessé ledit sieur de Bréval deux fois pendant la malladie, et que la veille de sa mort, il auroit prié ledit sieur curé de le recommander à la Saincte Vierge en laquelle il disoit avoir grand confiance $^{70}$ ". Timothée Le Hayer souhaite donc mourir dans la confession catholique : la peur de la mort l'incite à demander l'intercession de Marie et à revenir dans le giron catholique. La loi autorisant les curés à se présenter chez les protestants au seuil de leur mort pour les inciter à se convertir permet au clergé, semble-t-il, de remporter quelques succès. Toutefois, l'écuyer prévoyait que son abjuration serait difficile à accepter, notamment pour les membres de sa famille, et il avait prévenu le curé qu'il craignait " que sa femme et sa belle-mère, qui sont fort obstinées dans la RPR, ne fissent quelque scandale ${ }^{71}$ ". D'ailleurs, le prêtre indique qu'il " n'avoit ozé enteré [Timothée Le Hayer] ny à l'église ny au cimetière parce que quelques jeunes garçons l'en empeschèrent ${ }^{72}$ ». Certains membres de la communauté huguenote refusent donc le choix du sieur de Bréval embrassant la religion catholique à l'article de la mort. Ils veulent reprendre le corps du converti pour l'inhumer selon les dispositions calvinistes. L'abjuration de l'un des leurs est vécue comme un drame, une trahison et les réactions des huguenots sont violentes. Ils cherchent ainsi à empêcher les abjurations de leurs coreligionnaires. C'est à nouveau l'archidiacre qui relate ces événements survenus en 1670 et 1671 : "Henriette de Gallot, fille du sieur de Tilly, la nuit de la mort de son père, [auroit esté enlevée] par ceux du parti de la Religion Prétendue Réformée, et que deux garçons et deux filles du défunct sieur de Bréval auroient pareillement esté enlevés par ceux du parti de la RPR ${ }^{73}$. " Les deux pères sont décédés après s'être convertis au catholicisme et les autres membres de la communauté calviniste, notamment les mères des enfants, craignent que les enfants soient retirés des familles et élevés dans la religion catholique. Ils préfèrent alors

69. Gueneau, Y., Les protestants du Centre, op. cit., p. 227.

70. Arch. dép. d'Eure-et-Loir, G 844, registre de la visite archidiaconale du Dunois, effectuée le 19 septembre 1671.

71. Arch. dép. d'Eure-et-Loir, G 844, le 19 septembre 1671.

72. Arch. dép. d'Eure-et-Loir, G 844, le 19 septembre 1671.

73. Arch. dép. d'Eure-et-Loir, G 844, le 19 septembre 1671. 
prendre des précautions et cacher les enfants susceptibles d'être catholicisés. Cet exemple n'est pas unique : à Issoudun, les enfants d'un converti " sont envoyés en pension à l'extérieur d'Issoudun, peut-être Orléans, Paris ou une ville refuge ${ }^{74}$ ". Un arrêt du 16 juin 1681 interdit d'ailleurs aux protestants " d'user d'aucunes menaces, intimidations, artifices ou voyes de fait, pour empêcher la conversion desdits de la $\mathrm{RPR}^{75}$ ", preuve que ce type d'action était courant.

Pour conclure, la fin des années 1670 est synonyme d'affaiblissement de la communauté réformée. Elle ressent un besoin de protection qu'elle trouve auprès de la noblesse. C'est dans de telles conditions qu'intervient la Révocation de l'édit de Nantes le 18 octobre 1685. Quelques jours après, le roi prend la décision d'envoyer des troupes dans la région beauceronne afin de convertir les calvinistes par la force, la peur et la contrainte.

Dans la perspective de l'abandon et de la suppression du calvinisme, le logement des gens de guerre devient un moyen de pression destiné à obtenir l'abjuration des protestants avant qu'ils ne quittent leur domicile. Les troupes qui s'installent en Blaisois, en Beauce et dans le Perche sont utilisées dans les travaux de terrassement nécessaires à la construction d'un aqueduc à Maintenon, aqueduc destiné à approvisionner Versailles en eau puisée dans l'Eure. Les dragons interviennent à Blois, Mer, Lorges, Châteaudun, Bellême, Chartres et Dreux, mais " le déroulement exact des dragonnades n'est pas connu précisément ${ }^{76}$ " et aucune source locale n'atteste réellement la venue des troupes à Authon. Aucune trace d'abjuration collective n'a été enregistrée. Toutefois, la présence des militaires dans leur région, ainsi que la connaissance des exactions qu'ils commettent chez leurs voisins, incite probablement un certain nombre de réformés à abjurer, dans la crainte de voir approcher les dragons de leurs demeures. Les Églises réformées situées dans cette grande région parisienne sont pour Louis XIV, installé à Versailles en 1682, une provocation infligée à son pouvoir absolu. La présence massive de soldats au nord de Chartres est ainsi l'occasion d'une répression contre les dernières communautés huguenotes beauceronnes, résistantes mais affaiblies.

Et pourtant, la communauté protestante, ou du moins certaines de ses familles, survit à l'interdiction de son culte. En effet, en 1742 est ouvert un registre des décès de ceux privés de la sépulture catholique ${ }^{77}$, c'est-à-dire des protestants non convertis et des relaps. Quarante-quatre Authonais y figurent entre 1742 et 1787 . Ainsi, après une période d'apogée, l'Église s'affaiblit mais résiste à toute tentative d'extinction. Même si quelques familles demeurent calvinistes à la fin de l'Ancien Régime, la communauté réformée

74. Boisson, D., Les protestants de l'ancien colloque du Berry, op. cit., p. 137.

75. Ibidem, p. 108.

76. BoIsSon, D. et LIPPOLD, C., « Les protestants du centre de la France... ", op. cit., p. 372.

77. Arch. dép. d'Eure-et-Loir, registre des décès de ceux privés de la religion catholique, non coté. 
ne reste qu'un souvenir, comme peut l'écrire le géographe M. Doyen en 1786 : " Il y avoit autrefois beaucoup de protestants qui y avoient un prêche $^{78}$. " Mais c'est seulement après la Révolution française que le protestantisme s'éteint à Authon-du-Perche.

\section{RESUME}

La communauté protestante d'Authon-du-Perche, près de Chartres, apparaît dans nos sources en 1598, avec l'arrivée d'un pasteur. L'Église se maintient jusqu'à la révocation de l'édit de Nantes - en 1685 - et sa vie nous est principalement connue par son registre d'état civil et ses actes notariés. Nous découvrons alors une population en majorité riche et bourgeoise, ayant rapidement acquis une bonne culture religieuse et de nouvelles mours, synonymes d'une adhésion profonde à la nouvelle religion. Les calvinistes sont minoritaires au sein du bourg. Néanmoins, les archives ecclésiastiques catholiques soulignent les bonnes relations entre les membres des deux communautés, les liens qui les unissent et les efforts effectués pour limiter les tensions. Cependant, en fin de siècle, les rapports entre les deux groupes se dégradent à la faveur des choix politiques de Louis XIV, qui souhaite éradiquer les protestants de son royaume. La communauté se replie, sans toutefois disparaître complètement.

\section{ABSTRACT}

The protestant community of Authon-du-Perche, near Chartres, appears in our sources in 1598, with a minister's arrival. The Church holds until the Revocation of the Edict of Nantes - in 1685 - and we principally know its life by its register of births, marriages and deaths and its deeds. We find a majority of rich and middleclass population, who quickly acquired good religious knowledge and new habits, that means a real adherence to the new religion. The Calvinists are a minority within the village. Nevertheless, the catholic records emphasize the good relationships between members of the two communities, the bonds which unite them and the efforts made to limit tensions. However, in the end of the century, relations between the communities deteriorate because of Louis XIV's political choices : he wants to eradicate protestant people of his kingdom. So, the Calvinist community withdraws into itself, without disappearing totally yet.

78. Doyen, M., Histoire de la ville de Chartres, du pays chartrain et de la Beauce, Chartres, 1786, p. 322. 